\title{
Liderazgo docente en comunidades profesionales de aprendizaje: Estudio de caso de dos escuelas públicas en México
}

\author{
Teaching Leadership in Professional Learning Communities: A Case Study From Two Public \\ Schools in Mexico
}

\section{Liderança docente em comunidades profissionais de aprendizagem: Estudo de caso de duas escolas públicas no México}

Carmen Celina Torres-Arcadia

Tecnologico de Monterrey

Escuela de Negocios

Nuevo León, México ctorres@tec.mx

iD https://orcid.org/0000-0001-6983-0000

Recibido • Received • Recebido: 18 / 02 / 2019

Corregido • Revised • Revisado: 07 / 08 / 2020

Aceptado • Accepted • Aprovado: 19 / 11 / 2020

\begin{abstract}
Resumen: Este estudio tuvo como objetivo describir el liderazgo docente que ejercen profesoras y directivas en comunidades profesionales de aprendizaje, en dos escuelas primarias públicas. Desde el enfoque de investigación de casos multiples de corte cualitativo, se utilizó la observación naturalista y las entrevistas conversacionales. El desarrollo de las comunidades fue acorde con el modelo de aprendizaje organizacional, que comprende: organización inicial, identificación de problemática, adquirir conocimiento, compartir, usar y difundir conocimiento. Los resultados indican similitudes y diferencias en la participación de las profesoras y directivas en ambos casos. Entre las similitudes se encontró que la experiencia ayudó a las participantes a crecer y a aprender, y que es posible estimular el liderazgo y colaboración en el marco de una comunidad profesional de aprendizaje. Entre las diferencias, la disposición de las directivas fue notoria, mientras que en una de las escuelas todas las maestras participaron motivadas por la directora, en la otra escuela no se contó con el apoyo de las directivas y las profesoras participaron en forma espontánea. Es posible concluir que en ambas escuelas
\end{abstract}


http://doi.org/10.15359/ree.25-1.4

http://www.una.ac.cr/educare

educare@una.ac.cr

se observó el surgimiento del liderazgo docente al darles voz a las participantes en sus iniciativas, ideas, experiencias, creatividad y el reconocimiento al dominio personal. Finalmente, ante la resistencia e incertidumbre que estos procesos producen, se propone que estos temas de colaboración en CPA y de liderazgo distribuido se constituyan como pilares de la escuela y sean incorporados en la cultura escolar, así como en la formación inicial y continúa de personal docente y directivo.

Palabras claves: Liderazgo docente; comunidades profesionales de aprendizaje; profesorado de educación básica.

Abstract: This study aimed to describe the teaching leadership exercised by female teachers and principals in professional learning communities (CPA) in two public elementary schools. Naturalistic observation and conversational interviews were used under a qualitative multiple-case research approach. The development of the communities was in accordance with the organizational learning model, which includes initial organization, identification of problems, acquisition of knowledge, sharing, and use and dissemination of knowledge. The results show similarities and differences in the participation of the teachers and principals in both schools. Among the similarities, it was found that the experience helped the participants to grow and learn and that it is possible to stimulate leadership and collaboration within the framework of a professional learning community. Among the differences, the disposition of the principal was notorious. While in one of the schools, all the teachers participated, motivated by the principal, in the other school, they did not have the principal's support, and the teachers participated spontaneously. It is possible to conclude that, in both schools, the emergence of teaching leadership was observed by giving participants a voice in their initiatives, ideas, experiences, creativity, and recognition of personal mastery. Finally, given the resistance and uncertainty that these processes generate, it is proposed that the collaboration in CPA and distributed leadership topics become the pillars of the school and be incorporated into the school culture, as well as in the initial and continuous training of teachers and principals.

Keywords: Teacher leadership, professional learning communities, elementary school teacher.

Resumo: Este estudo teve como objetivo descrever a liderança de docentes exercida por professoras e a direção em comunidades de aprendizagem profissional, em duas escolas públicas de ensino fundamental. Sob o enfoque de pesquisa de casos múltiplos de abordagem qualitativa foi utilizada a observação naturalista e as entrevistas conversacionais. O desenvolvimento das comunidades obedeceu ao modelo de aprendizagem organizacional, que inclui: organização inicial, identificação de problemas, aquisição de conhecimentos, partilha, utilização e disseminação de conhecimentos. Os resultados indicam semelhanças e diferenças na participação de professoras e da direção, em ambos os casos. Entre as semelhanças, verificou-se que a experiência ajudou as participantes a crescer e aprender que é possível estimular a liderança e a colaboração no âmbito de uma comunidade de aprendizagem profissional. Entre as diferenças, a disposição da direção foi notória, enquanto em uma das escolas todas as professoras participaram motivadas pela diretora, na outra escola não tiveram o apoio da diretoria e as professoras participaram espontaneamente. É possível concluir que em ambas as escolas, o surgimento da liderança de docentes foi observada, dando voz as participantes em suas iniciativas, ideias, experiências, criatividade e reconhecimento do domínio pessoal. Por fim, dadas às resistências e incertezas que esses processos produzem, propõe-se que essas questões da colaboração na CPA e da liderança distribuída se tornem os pilares da escola e sejam incorporadas na cultura escolar, bem como na formação inicial e continuada de professores e diretoria.

Palavras-chave: Liderança docente; comunidades profissionais de aprendizagem; professor de educação básica. 
http://doi.org/10.15359/ree.25-1.4

\section{Introducción}

El liderazgo docente en contextos de comunidades profesionales de aprendizaje (en adelante (PA) ha cobrado relevancia en los últimos años. Aún y cuando los estudios sostienen que para la mejora educativa es importante reconocer al profesorado como profesionales con iniciativa, capaces de colaborar y de ejercer un liderazgo en conjunto con el personal directivo, estas manifestaciones se presentan aisladas en los contextos escolares sin formar parte de una cultura colaborativa. Al respecto, Carpenter (2015) señala que una cultura colaborativa es la forma en la que los maestros, maestras y personal administrativo piensan y se comportan al compartir información sobre su práctica. Una cultura de colaboración es el proceso sistemático en el que los grupos docentes, así como los administrativos usan para trabajar juntos, de manera interdependiente, con el propósito de analizar e impactar su práctica profesional y, con ello, mejorar el aprovechamiento de los alumnos y alumnas. Por otro lado, Harris y Jones (2017) sostienen que la idea de las CPA está respaldada por el concepto de liderazgo distribuido y sus beneficios; sin embargo, también señalan que no son la panacea, dado que es común que las escuelas enfrenten obstáculos como lo son: el mal uso del poder, la sobrecarga de trabajo y barreras que dificultan la conformación de una CPA y el liderazgo docente, aún así añaden que son oportunidades que pueden ser eficaces, si se realizan sabiamente.

En este artículo, se examina el proceso de formación de dos CPA integradas por profesoras y directivas de escuelas primarias públicas en la ciudad de Monterrey, Nuevo León en México. Las profesoras participaron identificando problemáticas en sus escuelas y plantearon soluciones. Para tener una comprensión clara de cómo se manifiesta la participación en las CPA se intentó estudiarlas desde las perspectivas del liderazgo docente y la comunidad profesional de aprendizaje basada en la colaboración y aprendizaje organizacional (Argyris, 2001; Harris, 2003; Senge, 2006).

Se entiende por liderazgo docente cuando el profesorado extiende su participación más allá de su aula de clases (Harris, 2003; Reeves, 2009; Spillane, 2006), ya que la función de liderazgo del profesorado en una escuela no se limita a quienes la ejercen de manera formal a través de algún puesto directivo, jefaturas o coordinaciones, sino también de aquel profesorado que, en forma voluntaria e informal, apoya a otros colegas, compartiendo su expertise y conocimiento, además que establecen iniciativas en su escuela, sin tener una posición formal de liderazgo.

Harris (2003) sustenta que cuando un maestro o maestra usa su experiencia para ayudar a sus colegas está actuando como un modelo a seguir. El liderazgo docente puede ser un factor influyente para la transformación de las prácticas de instrucción. La teoría del liderazgo docente destaca dos procesos para la formación y transformación docente: el liderazgo distribuido y la colegialidad.

El liderazgo distribuido se asocia con nociones de empoderamiento, trabajo en equipo, colegialidad, motivación del personal, desarrollo de capacidades, liderazgo docente, autonomía 
http://doi.org/10.15359/ree.25-1.4

http://www.una.ac.cr/educare

educare@una.ac.cr

profesional y desarrollo profesional. La colegialidad da como resultado una fuerte cultura profesional en la escuela, ya que incluye la colaboración entre docentes, el respeto mutuo, los valores compartidos de trabajo, la cooperación y diálogos sobre la enseñanza y el aprendizaje (Harris, 2003).

El concepto de CPA ha cobrado influencia en los campos de la administración, ciencias sociales $y$, en particular, en educación, donde se le atribuyen numerosos propósitos, pero la finalidad última de la CPA en el campo educativo es el cambio e innovación (Fullan, 2016). Este enfoque se caracteriza generalmente por sus metas y visiones comunes, el aprendizaje colectivo, la colaboración, el diálogo, los significados compartidos y, sobre todo, por la sensación de comunidad que toma lugar entre sus miembros (Dufour y Eaker, 1998; Wenger, 2001). En el campo educativo, la implementación de una CPA reduce el tradicional aislamiento e individualismo del profesorado (Lortie, 1974), e incrementa el compromiso y se genera una responsabilidad compartida con la misión, metas y cambio educativo de la escuela.

La bibliografía muestra que los resultados esperados de los sistemas educativos dependen en un alto grado del profesorado . Con ello, se espera que el rol docente se transforme de ser solo transmisor de conocimiento a líder que interviene y propicia acciones de cambio en sus escuelas en colaboración con sus colegas (Elizondo Huerta, 2010; Fullan, 2016; Hargreaves, 2005). En los casos que aquí se describen, se observa la manera en la que las profesoras y directivas, al organizarse en CPA, logran un ambiente propicio para la participación conjunta que favorece su liderazgo y, consecuentemente, el mejoramiento de sus prácticas.

En el nivel de educación básica en México funciona el ConsejoTécnico Consultivo (Secretaría de Educación Pública, 1982), el cual es un órgano colegiado integrado por la persona directora y profesorado, quienes se reúnen una vez al mes en cada escuela con el propósito de conducir acciones de planeación y toma de decisiones comunes enfocadas a cumplir la misión educativa con el liderazgo del director o directora. Aún cuando este consejo sea útil para la organización y tener posibilidades para estimular el liderazgo distribuido del profesorado y la colaboración, en la práctica existe todavía una distancia considerable para poseer las características distintivas de una CPA, entre las que se distinguen la participación y pertenencia voluntaria y colaboración entre otras. A este respecto, Hargreaves (2005) y Lortie (1974) coinciden en señalar que, a pesar de los esfuerzos por establecer la colaboración dentro de las escuelas, hay mucho que hacer, dado que el trabajo aislado y rutinario del profesorado persiste. A esto se añade la tradicional figura directiva y su ejercicio del poder. Sepúlveda y Aparicio (2017) citan que las "condiciones para que se dé un liderazgo distribuido son relativas, dado que la estructura formal y la figura del director o la directora siguen siendo centrales para la asignación de tareas y la resolución de conflictos" (p. 9). 
http://doi.org/10.15359/ree.25-1.4

Entre las alternativas para impulsar el trabajo colectivo, así como la participación y liderazgo del profesorado, se considera que la CPA es promisoria y valiosa para la gestión escolar dentro de la cual ocurren encuentros entre profesorado motivado por aprender, compartir y diseminar conocimiento producto de su experiencia y conocimiento tácito, así como la oportunidad de establecer diálogos reflexivos. Una CPA es, además, una fuente de innovación continua y promotora de cambio en un contexto de liderazgo distribuido que considere las voces e iniciativas de sus docentes en el mejoramiento de la práctica y calidad educativa de sus propias realidades.

La implementación de la CPA en una escuela no ocurre de la noche a la mañana; existe un proceso paulatino que implica esfuerzos, resistencias y la incorporación de una cultura colaborativa y menos aislada, como ocurre comúnmente en las escuelas (Lortie, 1974). En México existen algunos estudios y experiencias con el enfoque de CPA y colaboración. Entre ellos se encuentra el de Flores y Flores (2005), consistente en un estudio multicasos de escuelas en el noreste de México realizado en varias etapas, que describe el proceso de implementación de CPA, así como la investigación de Martínez-Cruz y Moreno-Olivos (2017) quienes realizaron un análisis de la colegialidad docente en la escuela primaria mexicana.

En la siguiente sección se intenta ampliar los conceptos de liderazgo y comunidad de aprendizaje, así como los vínculos que existen entre estos y las características que comparten.

\section{Marco teórico}

En la literatura internacional se destacan definiciones de liderazgo docente de diferentes estudios del tema, que van desde la capacidad de animar a colegas a cambiar, y hacer cosas que normalmente no consideran sin la influencia del líder (Wasley, 1991), así también que los maestros y las maestras que realizan acciones de líderes, dentro y fuera del aula, se identifican y contribuyen con una comunidad educadora y líder e influyen en la mejora de la práctica educativa (Katzenmeyer y Moller, 2009); mientras que Boles (1992) lo contrasta con las nociones tradicionales de liderazgo, al caracterizarlo como una forma de liderazgo colectivo en el que los maestros y las maestras desarrollan su experiencia trabajando en colaboración. Estas definiciones son consistentes con lo referente a las características de las CPA y al aprendizaje organizacional.

Day y Harris (2003) sugieren que hay cuatro dimensiones de la función de liderazgo docente. La primera se refiere a la traducción de los principios de mejora de la escuela en las prácticas de las aulas individuales. La segunda se centra en el liderazgo participativo, donde todo el profesorado se siente parte del cambio o desarrollo y tiene un sentido de propiedad, trabaja con sus colegas para dar forma a los esfuerzos de mejora de la escuela y tomar un poco de liderazgo en la orientación del profesorado hacia una meta colectiva. La tercera dimensión para el mejoramiento escolar es el rol mediador. Finalmente, la cuarta y posiblemente la dimensión 
http://doi.org/10.15359/ree.25-1.4

http://www.una.ac.cr/educare

educare@una.ac.cr

más importante es forjar relaciones estrechas con sujetos individuales a través de las cuales el aprendizaje mutuo tiene lugar.

Se considera al liderazgo distribuido como la capacidad del personal docente y administrativo de aprender en un ambiente de colaboración (Lattimer, 2007). Así también constituye una teoría emergente cuyo propósito es ofrecer nuevas perspectivas del liderazgo escolar. El liderazgo distribuido dentro de una escuela puede permitir cambios en la base de conocimientos de los maestros y las maestras, así como en las prácticas de enseñanza, ya que puede tener un impacto positivo en el logro del estudiantado (Harris, 2003). A partir de esto se considera que el liderazgo distribuido es un proceso de influencia compartida en el que participan y contribuyen varios individuos, el cual surge cuando estos interactúan y juntos forman un grupo de redes en las que la experiencia esencial es compartida.

Los inicios de las CPA se remontan a los años 80, cuando Rosenholtz (1989) señaló la importancia que tienen las condiciones del lugar de trabajo en la discusión sobre la calidad de la enseñanza. Este autor sostuvo que los profesorados que se sentían apoyados en su propio aprendizaje y práctica en el aula eran más comprometidos y eficaces que aquellos que no recibían estos apoyos. Las redes de docentes, la cooperación entre colegas y la ampliación de los roles profesionales aumentaron la eficacia de los maestros y las maestras para satisfacer las necesidades del estudiantado. Lo anteriormente señalado provee un ambiente colaborativo y democrático que estimula el liderazgo distribuido del profesorado.

Rosenholtz (1989) encontró que cuando los maestros y las maestras tenían oportunidades para la investigación colaborativa y el aprendizaje relacionado con ella, eran capaces de desarrollar y compartir un cuerpo de sabiduría extraído de su propia experiencia. Harris y Jones (2017) sostienen que la CPA es una estrategia para mejorar el sistema escolar; DarlingHammond (1996) destacó que la toma de decisiones compartidas es un factor importante de transformación y cambio de los roles de enseñanza en las escuelas, ya que se proporciona tiempo estructurado para que los maestros y las maestras trabajen juntos en la planificación de la instrucción, observando las aulas de los demás y compartiendo comentarios. La bibliografía sobre las CPA brinda, repetidamente, atención a cinco atributos esenciales: liderazgo solidario y compartido, creatividad colectiva, valores y visión compartidos, condiciones de apoyo y práctica personal compartida (Darling-Hammond, 1996; Harris y Jones, 2017).

En las escuelas, la comunidad de aprendizaje puede formarse por personas de diversos y múltiples grupos, en todos los niveles, que colaboran y trabajan continuamente en conjunto (Louis y Kruse, 1995). Tal trabajo colaborativo se basa en lo que Louis y Kruse (1995) etiquetan como diálogo reflexivo, en el cual el profesorado sostiene conversaciones e identifica problemas sobre el estudiantado, la enseñanza y el aprendizaje. 
http://doi.org/10.15359/ree.25-1.4

Los estudios aquí citados concuerdan en que el liderazgo distribuido es un factor clave en el mejoramiento, cambio y desarrollo de la escuela como organización escolar, a la vez abogan en que este tipo de liderazgo contrasta con liderazgos verticales por mandato, que residen en el poder de los grupos directivos, por lo que se hace necesario un liderazgo co-construido entre doentes. Lo anterior puede ser posible en espacios como las CPA, las cuales propician oportunidades para el desarrollo profesional docente, al establecer diálogos profesionales que permiten el aprendizaje y construcción de significados, así como la generación, diseminación y aplicación de conocimiento en forma colaborativa.

En lo anteriormente señalado se aboga por una reconceptualización del liderazgo que supere ser solo un slogan y se convierta en la acción en un componente esencial de una gestión innovadora en la cual personal directivo y docente compartan sus visiones compartidas que promuevan la calidad educativa de las escuelas.

Los casos de dos escuelas que se presentan a continuación constituyen intervenciones en un campo poco explorado para organizar y mejorar las escuelas, así como para ofrecer datos y reflexiones generadas a través de la investigación, que puedan ser aplicados a una gestión innovadora que involucre a los grupos directivos y profesorados en acciones conjuntas y democráticas.

\section{Metodología}

Se utilizó el enfoque de casos múltiples (Yin, 2017), el cual permitió registrar y comparar los eventos que ocurrieron en las dos escuelas mientras su profesorado participaba en una CPA durante enero-diciembre 2011 y enero-junio 2012. Cada una de las escuelas fue analizada como un caso independiente desde una perspectiva cualitativa, con descripciones literales thick description a través de las observaciones participantes y entrevistas, lo cual coloca a las personas investigadoras como las principales recolectoras de datos a fin de producir una narrativa ricamente descriptiva de los casos (Merriam, 2015).

Las escuelas fueron seleccionadas con base en los criterios de que las personas directivas y profesorado participaran de manera voluntaria y que, además, fueran escuelas "promedio", es decir, que no fueran escuelas que tuvieran excelente reputación, ni tampoco escuelas con serios problemas con alumnado o con profesorado problemático. Cabe señalar que ninguna de las participantes tenía formación en liderazgo ni había participado en una CPA. Para proteger la confidencialidad de las participantes y de las escuelas se siguió la imposición de anonimato descrito por Gibbs (2014), por lo cual se omitieron los nombres reales y se asignaron nombres ficticios. En la Tabla 1 se presentan algunos datos de las profesoras y directoras participantes de los dos casos estudiados, los cuales se denominan: Caso: Escuela Leona Vicario y Caso: Escuela Vicente Guerrero. 
http://doi.org/10.15359/ree.25-1.4

http://www.una.ac.cr/educare

educare@una.ac.cr

Tabla 1: Las participantes del estudio

\begin{tabular}{|c|c|c|c|c|c|c|c|}
\hline \multicolumn{4}{|c|}{ Escuela Leona Vicario } & \multicolumn{4}{|c|}{ Escuela Vicente Guerrero } \\
\hline \multicolumn{2}{|c|}{ Profesora } & \multirow{2}{*}{$\frac{\text { Experiencia }}{25 \text { años }}$} & \multirow{2}{*}{$\frac{\text { Grado }}{1^{\circ} \mathrm{A}}$} & \multicolumn{2}{|c|}{ Profesora } & \multirow{2}{*}{$\begin{array}{c}\text { Experiencia } \\
23 \text { años }\end{array}$} & \multirow{2}{*}{$\frac{\text { Grado }}{5^{\circ}}$} \\
\hline Silvia & (PLV1) & & & Margarita & (PVG1) & & \\
\hline María & (PLV2) & 18 años & $5^{\circ}$ & Alejandra & (PVG2) & 20 años & $6^{\circ}$ \\
\hline Ana & (PLV3) & 21 años & $6^{\circ}$ & Carolina & (PVG3) & 4 años & $2^{\circ}$ \\
\hline Laura & (PLV4) & 19 años & $3^{\circ}$ & Dora & (PVG4) & 1 año & $4^{\circ}$ \\
\hline Julia & (PLV5) & 2 años & $2^{\circ}$ & & & & \\
\hline Blanca & PLV6) & 3 años & $1{ }^{\circ} \mathrm{B}$ & & & & \\
\hline Deyanira & (PLV7) & 7 años & $4^{\circ}$ & & & & \\
\hline Directora & (DLV) & 20 años & & Directora & DVG) & 27 años & \\
\hline
\end{tabular}

Nota: Elaboración propia. Los nombres de las escuelas, profesoras y directivas participantes son ficticios.

Dado que el equipo investigador estuvo inmerso en el proceso de las CPA, se utilizó la observación naturalista con participación moderada (Merriam, 2015) y las entrevistas conversacionales (Mason, 2018). La participación moderada ocurre cuando quien investiga busca mantener un balance entre los roles de adentro y afuera -insider y outsider- en las actividades de participación y observación, esto significa que las personas investigadoras al involucrarse eventualmente en la escuela, pasan a ser miembros del proceso de las comunidades, pero también son externas a esta (Merriam, 2015). En cuanto a las entrevistas, estas fueron abiertas y tomaron la forma de conversaciones con las profesoras y directoras durante el transcurso del proceso, de acuerdo con Mason (2018), este tipo de entrevistas son las adecuadas para conocer y monitorear lo que sucede desde las perspectivas de las participantes.

Para Lincoln y Guba (1985) y Erlandson, Harris, Skipper y Allen (1993), los criterios para construir validez en un estudio cualitativo son: credibilidad, transferibilidad, dependencia y confirmabilidad, los cuales contienen las técnicas para la validación de un estudio. En esta investigación se aplicaron las técnicas correspondientes a dichos criterios (véase Tabla 2).

Tabla 2: Criterios y técnicas de validación

\begin{tabular}{ll}
\hline \multicolumn{1}{c}{ Criterio } & \multicolumn{1}{c}{ Técnica } \\
\hline Credibilidad & $\begin{array}{l}\text { Triangulación } \\
\text { Member checking }\end{array}$ \\
Transferibilidad & Selección de participantes con base en criterios \\
Dependencia & Diario reflexivo \\
Confirmabilidad & Diario reflexivo \\
& Member checking \\
\hline
\end{tabular}

Nota: Adaptado de Erlandson et al. (1993) y Lincoln y Guba (1985). 
http://doi.org/10.15359/ree.25-1.4 http://www.una.ac.cr/educare educare@una.ac.cr

La técnica de validación member checking para dar credibilidad y confirmabilidad al estudio consistió en verificar con sus participantes los datos provenientes de las entrevistas y observaciones durante la investigación (Erlandson et al., 1993), así, también se utilizó la triangulación de datos, la cual mostró que un determinado resultado obtenido por medio de la entrevista se presenta también en las observaciones, o en la entrevista con otra persona, para así dar apoyo o reforzar dicho resultado (Lincoln y Guba, 1985). Para la transferibilidad se utilizó la técnica de selección de los participantes con base en criterios, con respecto a la dependencia y confirmabilidad se utilizó el diario reflexivo de los sujetos investigadores (Erlandson et al., 1993).

La Figura 1 muestra la estructura metodológica de la CPA que orientó las acciones de las profesoras participantes, la cual se basó en el modelo de aprendizaje organizacional (AO), derivado de los trabajos de DiBella y Nevis (1998); Senge (2006) y Wenger (2001).

Figura 1: El modelo de aprendizaje organizacional

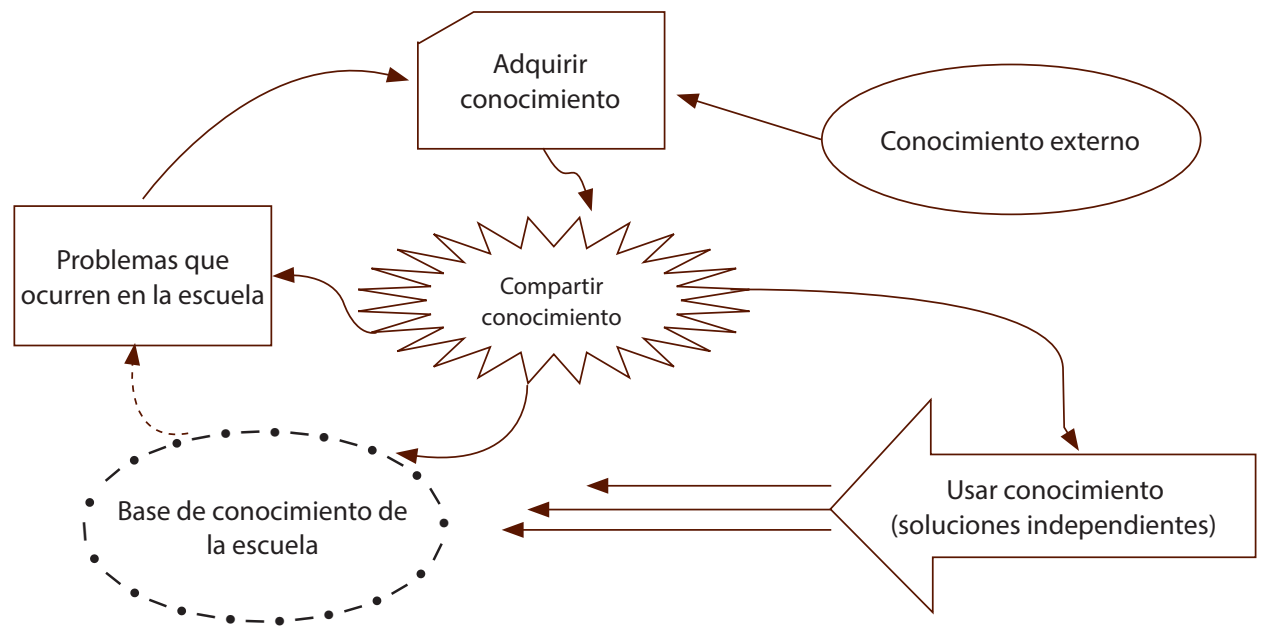

Nota: Adaptado de DiBella y Nevis (1998); Senge (2006) y Wenger (2001).

Este modelo teórico $(\mathrm{AO})$ constituyó el marco que sustenta que en toda organización escolar existe una base de conocimiento de profesorado y personal directivo, que puede ser utilizada cuando estos grupos identifican problemas que ocurren en la escuela y desean resolver con una CPA. En estos casos se estableció una dinámica en la cual sus miembros aprenden entre sí acerca del problema, a la vez que adquieren conocimiento externo mediante el diálogo e interacción para su solución; tienen la oportunidad de compartir conocimiento y experiencias a través del intercambio colaborativo $y$, consecuentemente, usar ese conocimiento. De esta manera, de acuerdo con DiBella y Nevis (1998), Senge (2006) y Wenger (2001), ocurre un proceso de $\mathrm{AO}$ a través de interacciones sociales a nivel de grupos, en el cual la organización se 
http://doi.org/10.15359/ree.25-1.4

http://www.una.ac.cr/educare

educare@una.ac.cr

adapta a entornos cambiantes al generar conocimiento útil que puede ser usado al compartirlo y diseminarlo para el mejoramiento de la organización.

\section{Los casos de las escuelas participantes}

Los resultados se organizaron con base en el contexto, las características de las participantes, la organización de la CPA y las fases del aprendizaje organizacional implementadas en cada una de las escuelas.

\section{Caso: Escuela Leona Vicario}

El contexto. Esta escuela trabajaba en la modalidad de horario extendido, en la cual sus estudiantes asistían tanto en el turno matutino como en el vespertino, pero con con personal directivo y profesorado diferentes en ambos turnos. La directora del turno matutino aceptó la invitación del equipo investigador de que las profesoras de este turno participaran en el proyecto durante el ciclo escolar enero-junio 2011.

La escuela, ubicada en un sector de estrato socioeconómico bajo, se consideró de dimensión pequeña, pues contaba con siete grupos atendidos por siete profesoras. El tamaño de los grupos que atendían estas profesoras era de 19 a 35 estudiantes. Esta escuela participó en diversos proyectos promovidos por el gobierno del Estado y la Secretaría de Educación, en los cuales habían obtenido primeros lugares.

Las características de las participantes. La directora era una persona reconocida por las maestras. Ella se hizo cargo de la escuela en un momento que la institución atravesaba por un período difícil, ya que había una amenaza de cierre por baja demanda de matrícula, además de una pobre reputación de la escuela, dado que la población escolar provenía de la expulsión de otras escuelas, por mala conducta. La escuela entró a la modalidad de horario extendido lo cual, en opinión de las profesoras, fue visto por los padres y madres de familia como una guardería. La escuela inició en un proceso paulatino de cambio a través de la implementación de diferentes proyectos y campañas con la niñez y padres y madres de familia, el cambio era palpable y sentido en opinión de las profesoras.

En cuanto a las características de las profesoras que aceptaron participar animadas primordialmente por la directora, cuatro tenían antigüedad en la profesión docente, mientras que las restantes tres profesoras eran jóvenes principiantes. Las profesoras en general parecían mostrar interés en el proyecto. Las de mayor antigüedad eran más comunicativas, mientras que las profesoras con menos experiencia se mostraban a la expectativa, hablaban poco y se limitan a escuchar a sus compañeras.

Entre las profesoras de mayor experiencia destacaba Silvia, quien atendía el 1 er grado "A". Ella, además de ser docente, había trabajado en programas de capacitación de profesorado de la Secretaría de Educación. María, la profesora del $5^{\circ}$ grado, era otra profesora con experiencia, 
http://doi.org/10.15359/ree.25-1.4

y disposición por aprender y compartir su práctica docente. Ana, quien se autodenominaba la decana de la escuela, tenía a su cargo el $6^{\circ}$ grado, había permanecido en la escuela durante casi toda su carrera docente de 21 años, por lo que era considerada testigo tanto del desarrollo como de los problemas de la escuela. Laura, la profesora de 3er grado, era muy comunicativa, participativa y con voluntad a compartir su experiencia.

En cuanto a las profesoras más jóvenes, Julia recientemente había terminado sus estudios en la Escuela Normal, ella se mostraba reservada y tímida, pero sorprendía en ocasiones por su interés en platicar sobre su práctica de enseñanza en su aula de $2^{\circ}$ grado. Blanca atendía al $1^{\text {er }}$ grado "B" como profesora suplente y estaba en espera de una plaza segura. Deyanira recién se había incorporado a la escuela, comentó que anteriormente había trabajado en una escuela conflictiva enclavada en una colonia de nivel socioeconómico bajo.

Primera fase: organización de una comunidad de aprendizaje. Esta fase consistió básicamente en organizar la comunidad de aprendizaje, identificar los problemas a resolver, establecer horarios y formas de trabajar. Es importante señalar que esta fase inicial fue percibida por las profesoras como incierta, ya que existían dudas de cómo se desarrollaría la comunidad en la práctica.

El equipo investigador les solicitó que identificaran algún problema de su práctica educativa que quisieran comprender y resolver en la comunidad de aprendizaje. La directora y profesoras decidieron que el tiempo que dedicarían a la comunidad sería de cuarenta y cinco minutos de 11.45 a 12.30 a.m. los días martes y miércoles. En un inicio las siete profesoras estuvieron de acuerdo en que el problema a resolver era la comprensión lectora. Sin embargo, al discutir este problema entre ellas y con el equipo investigador surgieron otros problemas que consideraban aún más prioritarios como la indisciplina, adolescencia temprana y precocidad.

Posteriormente se llegó a la decisión de crear dos grupos para resolver los problemas seleccionados. El primer grupo quedó conformado por las profesoras Silvia, Blanca, Julia y Laura de los grados de $1^{\circ} \mathrm{A}$ y $\mathrm{B}, 2^{\circ}$ y $3^{\circ}$, decidieron trabajar con los problemas de indisciplina y desintegración familiar y el segundo grupo lo conformaron las profesoras Deyanira, María y Ana de $4^{\circ}, 5^{\circ}$ y $6^{\circ}$ grados interesadas en la problemática de adolescencia temprana, precocidad y violencia. Cada grupo tendría una reunión semanal de 45 minutos.

Segunda fase: adquirir conocimiento. Esta fase estuvo dedicada a que cada equipo aprendiera más acerca de las problemáticas de su interés. Las profesoras en conjunto con el equipo investigador estuvieron leyendo artículos y reportes de investigaciones con las temáticas de violencia, indisciplina, desintegración familiar, precocidad sexual, con el objetivo de que ellas incrementaran su comprensión y aprendieran más del tema. Sin embargo, fue notorio el hecho de que las profesoras le adjudicaban más valor a sus experiencias que a las aportaciones teóricas de los artículos consultados. 
http://doi.org/10.15359/ree.25-1.4

http://www.una.ac.cr/educare

educare@una.ac.cr

Con respecto al proceso de adquirir conocimiento llevado en esta fase, la profesora Silvia dijo: "Este espacio nos hace compartir y enterarnos de lo que sucede y ayudarnos y aconsejarnos entre todas" (PLV1). Por su parte la profesora Ana comentó:

Este proyecto se me ha hecho ligero, o a lo mejor algunas de las compañeras pueden decir que es lento o que es tedioso, siempre comentar, pero muchas veces no nos damos tiempo para comentar sobre la práctica. (PLV3, testimonio, 16 febrero 2011)

Durante esta etapa fue notorio el cambio de esquemas mentales de las participantes, al ver las problemáticas identificadas en sus equipos de trabajo desde otras perspectivas, así como el reconocimiento de la utilidad de las reuniones para discutir y aprender, con lo cual se fueron eliminando algunas ideas y temores acerca de que la CPA es un proceso lento y tedioso e implica sobrecarga de trabajo a la docencia. Esto es importante, porque confirma la idea de Senge (2006) acerca de que la revisión y cambio de los esquemas mentales es indispensable para adquirir nuevos conocimientos.

Tercera fase: compartir el conocimiento. Durante esta fase las profesoras estuvieron compartiendo con sus colegas sus comprensiones y aprendizajes del tema, así como sus habilidades e intereses. Aquí resaltó el compartir estrategias aprendidas desde su experiencia. Estas estrategias eran relativas a los temas tratados en cuanto a la indisciplina en el aula y la adolescencia temprana.

Lo relevante en esta etapa fue que las profesoras con más experiencia compartían a las profesoras principiantes aquellas estrategias que en su práctica les habían resultado útiles. Durante esta fase fue notorio el reconocimiento al dominio personal (Senge, 2006). Esto implica reconocer la experiencia de otra profesora y además poner en práctica sus consejos y sugerencias, esto es un rasgo de liderazgo docente. Por ejemplo, en cuanto a las estrategias para tratar la indisciplina en el salón de clases, Julia mencionó al respecto:

Amíme han servido las estrategias que nos compartela maestra Silviaya que como uno apenas va saliendo de la carrera, a veces se cicla y puede pensar uno que a lo mejor no funciona tal o cual estrategia, pero si probamos nuevas no sabremos el resultado, pero ahora que hay un tiempo para compartir y aplicar esto, se puede hacer más. (PLV5, testimonio, 13 abril 2011)

Blanca comentó:

A mí me gustaría una junta general del consejo técnico así puedo escuchar las experiencias de mis compañeras, ver qué aprendizajes hay para aplicar en el aula, las estrategias me han servido para solucionar algunos problemas, hay unas que no he usado todavía, pero estoy segura que me serían de utilidad. (PLV6, testimonio,13 abril 2011) 
http://doi.org/10.15359/ree.25-1.4

Esta dinámica presentada en las CPA demostró el valor que las maestras le conceden al compartir conocimiento y su uso. Fue notorio nuevamente la manifestación del liderazgo docente y el aprendizaje organizacional al compartir en forma colectiva el conocimiento en beneficio de la escuela (Senge, 2006).

Cuarta fase: utilizar y difundir conocimiento: El propósito de esta fase fue utilizar el conocimiento y generar posibles soluciones basándose en el conocimiento adquirido en la CPA. En esta etapa las profesoras principiantes estuvieron aplicando las estrategias sugeridas por sus compañeras expertas. Dado que en esta etapa, además de compartir su experiencia y conocimientos, fue necesario difundir la experiencia adquirida en el desarrollo de la CPA, se generaron dos artículos de corte profesional elaborados por ellas mismas y asesoradas por el equipo investigador. Estos artículos se titularon: Adolescencia temprana en las escuelas primarias de educación pública, elaborado por las profesoras de $4^{\circ}, 5^{\circ}$ y $6^{\circ}$ grados y Compartiendo estrategias para lograr una mejor disciplina en el aula de educación básica, elaborado por las profesoras Blanca, Silvia, Julia y Laura de $1^{\circ} \mathrm{A}$ y $\mathrm{B}, 2^{\circ}$ y $3^{\circ} \mathrm{grado}$. Con estos artículos las profesoras difundieron su experiencia acerca de la forma en la que trataron de resolver los problemas identificados al inicio de la comunidad para que pudiese ser utilizada por otro profesorado interesado.

Estas actividades fueron llevadas a cabo en el semestre enero-junio como estaba planeado inicialmente, sin embargo, por iniciativa de las profesoras, el trabajo continuó en el semestre de agosto-diciembre con la misma metodología de trabajo y con las mismas comunidades. En agosto el proyecto que, en forma unánime, seleccionaron los dos grupos de trabajo fue el de comprensión lectora y un programa de valores, los cuales fueron llevados a cabo a través de un proyecto de currículo integrado en el que también involucraron la participación de los padres y madres de familia. El producto final fue un video para compartir su experiencia con otro profesorado.

\section{Caso: Escuela Vicente Guerrero}

El contexto. Durante el mes de enero de 2012 se invitó a personal docente y directivo de esta escuela primaria, ubicada al sur de la ciudad de Monterrey, Nuevo León, a integrar una CPA. En general la respuesta de docentes fue de molestia y de rechazo, mencionaron que no contaban con el tiempo para participar, ya que atendían otros proyectos de la Secretaría de Educación. Al término de la reunión y ante el casi intento fallido, cuatro profesoras manifestaron su disposición de participar. La aceptación para involucrarse en una CPA es un rasgo muy importante, ya que esta tiene un carácter plenamente voluntario.

La escuela contaba con doce grupos de primero a sexto grado, los cuales eran atendidos por once profesoras y un profesor, y administrada por una directora y una subdirectora, por la cantidad de grupos y docentes se consideraba de dimensión grande. La escuela está situada en un sector de estrato socioeconómico de clase media, a la cual asistían por lo general hijos de profesionistas. 
http://doi.org/10.15359/ree.25-1.4

http://www.una.ac.cr/educare

educare@una.ac.cr

Las características de las participantes. En el grupo de estas cuatro profesoras, destacaba un común denominador: su entusiasmo para trabajar, por cambiar y mejorar la situación de su escuela y de aprender cosas nuevas. Dos de las profesoras eran veteranas en la docencia, mientras que las otras dos eran principiantes, lo que hacía un grupo interesante integrado con expertas y novatas. Alejandra, una de las profesoras con experiencia, enseñaba en $6^{\circ}$ grado tenía 35 estudiantes. Ella se distinguía por ser muy extrovertida, entusiasta y proactiva, además de comunicativa y crítica con respecto a la docencia. Podría decirse que era la líder del grupo y fue la primera en decir"estoy interesada en participar en la comunidad de aprendizaje" (PVG2). Margarita, tenía a su cargo el $5^{\circ}$ grado el cual estaba compuesto por 29 estudiantes, recientemente había ingresado a trabajar en esta escuela, pero anteriormente había ya trabajado en otras escuelas. Comentó que en la escuela anterior en la que laboraba, a diferencia de la actual, el profesorado era más participativo y dispuesto a compartir ideas.

Dora, recién egresada de la Escuela Normal, era maestra suplente en el $4^{\circ}$ grado, el cual se componía de 38 estudiantes, al respecto,ella dijo tener algunas dificultades por el tamaño del grupo. Se mostraba muy dispuesta a aprender y pensaba que las ideas por compartir en el equipo le serían muy útiles en su práctica en el aula. Carolina, era una profesora muy joven, trabajaba en dos turnos: el turno de la mañana en esta escuela y el de la tarde en otra, se caracterizaba por ser muy comunicativa a la vez que receptiva y tenía a su cargo el $2^{\circ}$ grado con 34 estudiantes. La directora, aun cuando aceptó la integración de la CPA en la escuela, se mostró más preocupada por cuestiones burocráticas de la Secretaría de Educación, por lo que su participación y apoyo fue muy escaso, al igual que el de la subdirectora.

Primera fase: organización de una comunidad de aprendizaje. En la primera sesión se les solicitó a las profesoras que identificaran un problema que desearan resolver o un tema a tratar que estuviera relacionado con su práctica educativa, así como el establecer un plan de acción, horarios y lugar de reunión. Las profesoras externaron preocupaciones por la enseñanza de diferentes asignaturas, tales como las matemáticas y la lectoescritura. Sin embargo, dado que eran profesoras de diferentes grados fue difícil atender problemáticas específicas de estos grados. Un punto común que aglutinó los intereses de las cuatro integrantes fue "saber más acerca de estrategias cognitivas de enseñanza" (PVG1, 2, 3, 4).

Se hicieron acuerdos en cuanto horario y lugar para la reunión. Las cuatro profesoras convinieron en que las reuniones serían de una hora semanal de 9.00 a 10.00 a.m. los miércoles. El lugar de reunión fue la sala de computación. En cuanto a los horarios fue notorio como ellas mismas se organizaron, ya que dejaban a sus grupos con tareas en la hora asignada a la reunión. Alejandra solicitaba a una madre de familia voluntaria le atendiera a su grupo.

En cuanto a la dinámica de la reunión y dado que estaban interesadas en conocer acerca de estrategias de enseñanza aprendizaje cognitivas y aplicarlas en sus clases, se llegó al acuerdo de que este sería el eje temático de la comunidad. El equipo investigador les propuso que en cada 
http://doi.org/10.15359/ree.25-1.4

reunión se revisaran algunas estrategias del modelo dimensiones del aprendizaje de Marzano et al. (2011), esto tomaría la forma de un mini taller en la práctica. Se optó por este modelo, ya que las estrategias podrían ser fácilmente aplicadas en su propio contexto, además de ser enriquecidas con la experiencia de cada una. Así también las profesoras podrían compartir con el grupo el funcionamiento de las estrategias en la realidad de su salón de clases.

A cada profesora se le proporcionó una carpeta con diferentes secciones: una para coleccionar cada una de las estrategias del modelo dimensiones del aprendizaje, que el equipo investigador llevó impresas a cada una de las reuniones, una sección para anotaciones y la última para que registraran sus propias reflexiones del proceso.

Segunda fase: adquirir conocimiento. Esta etapa prácticamente se inició con la primera lección de las cinco dimensiones que integran el modelo de Marzano et al. (2011) la cual se compone de estrategias para crear un ambiente en el aula tanto de carácter emocional como para la tarea y se propicien actitudes positivas para el aprendizaje, con reglas claras para el comportamiento y el trabajo. Esta dimensión provocó en las profesoras mucho interés y constituyó un detonador para hablar de su práctica educativa, aprender y compartir, ya que fue enriquecida con base en su propia experiencia. Las profesoras con mayor antigüedad en la docencia fueron quienes estuvieron aportando ideas, procedimientos y otras estrategias adicionales que les habían funcionado. Por ejemplo, Alejandra comentó:

Me parece muy interesante lo que el modelo dice. Con base en mi experiencia el tener confianza a los alumnos, hace que ellos vean de otro modo a los maestros. Con respecto a los permisos en mi salón, quien quiera ir al baño, puede salir sin preguntar, solamente tiene que pararse ir al baño y regresar. (PVG2, testimonio, 15 febrero 2012)

Durante esta fase, las profesoras estuvieron aprendiendo la importancia de crear un ambiente adecuado de confianza, orden y aceptación en su clase como base para el aprendizaje. La exposición de las estrategias en el mini taller detonó en las profesoras muchas inquietudes que tenían con respecto al control de grupos, sus conductas y desempeños, lo que ocasionó que las conversaciones fueran extensas, y en ocasiones se desviaban a otros temas. En el transcurso de estas sesiones se notó la emergencia del reconocimiento al dominio personal y del liderazgo docente que, de acuerdo con Senge (2006), son facilitadores para el aprendizaje. Por ejemplo, Margarita en una reunión reconoce el talento de Alejandra, comentó:

Yo siempre le he echado muchas porras a Margarita desde que la conocí, cuando yo llegué a la junta de consejo yo veía que Margarita era la líder, ideas buenas, pero no la secundan en la escuela. (PVG1, testimonio, 22 febrero 2012) 
http://doi.org/10.15359/ree.25-1.4

http://www.una.ac.cr/educare

educare@una.ac.cr

Esta fase transcurrió aproximadamente en cuatro sesiones, aunque fue difícil establecer cuándo terminó una e inició otra, ya que en la práctica ocurren simultáneamente. Sin embargo, esta fase es importante en la medida que se aprende del problema o tema en cuestión y es un momento fundamental para que ocurran las siguientes etapas. En este caso las profesoras estuvieron aprendiendo y reflexionando acerca de sus propias prácticas docentes.

Tercera fase: compartir conocimiento. Esta fase transcurrió de una manera productiva. Las profesoras estuvieron compartiendo su experiencia con las estrategias, además de compartir otras que surgían en las conversaciones en la CPA tales como: formatos para el registro de cumplimiento de tareas y el buzón de la amistad. A continuación, se presentan algunos ejemplos de comentarios de las profesoras al momento de compartir: Margarita comentó a sus compañeras acerca de su preocupación por el incumplimiento de tareas de nueve estudiantes, así como el no saber qué hacer para que cumplan. Alejandra le compartió un formato que le había resultado efectivo:

Te sugiero que hagas un formato donde cada niño debe de llenar las tareas que se le encargan y la maestra lo debe firmar. En caso de que el niño no cumpla, el papá debe firmar de enterado y al día siguiente el niño debe traer lo que haya faltado más lo que se encargó ese día (PVG2, testimonio, 21 marzo 2012).

Carolina señaló tener problemas con la disciplina cuando los niños y las niñas regresan del recreo. Margarita le sugirió ponerles ejercicios de relajación, ya que les ayudaría a la concentración en clases. Durante esta etapa, también compartieron ideas para elaborar mapas mentales en las clases que impartían, lo cual correspondía a una de las estrategias de organización del conocimiento de la teoría de Marzano et al. (2011). Las maestras llevaron consigo a las reuniones de la CPA los mapas mentales de historia, matemáticas y geografía elaborados por sus estudiantes en sus clases.

Cuarta fase: utilizar y difundir conocimiento. En esta fase llegó el momento de diseminar el uso del conocimiento que habían compartido. La profesora Dora comentó en una de las reuniones haber tenido problemas con el trabajo en equipo de su grupo, hasta que sus compañeras le sugirieron integrar al alumnado en equipos de tres. En una reunión posterior comentó haber seguido las recomendaciones sugeridas por sus compañeras en su clase y que sí le habían funcionado.

Tres semanas más tarde, Margarita dijo que utilizó el registro que había compartido Alejandra y encontró que es un buen recurso, puesto que logró buenos resultados, ya que casi todos su alumnado estaban haciendo las tareas: 
http://doi.org/10.15359/ree.25-1.4

Me gustó mucho poder compartir con mis compañeras los pocos o muchos conocimientos que tengo sobre nuestra labor ya que esto nos enriquece y podemos ser mejores profesoras que es lo que los alumnos necesitan para ser personas valiosas para la sociedad. (PVG1, testimonio, 23 mayo 2012)

También Dora comentó haber utilizado el formato con algunas modificaciones que realizó de acuerdo con sus necesidades:

Este proyecto me trajo muchos beneficios ya que yo estaba recién llegada a la escuela y el compartir conocimientos con otras maestras me ayudó a realizar mi trabajo de una mejor manera. (PVG4, testimonio, 23 mayo 2012)

En esta fase, las profesoras dialogaron acerca de la manera en la que podrían difundir a otros profesores y profesoras la experiencia del semestre y pensar en un producto final de cierre del trabajo realizado en la CPA. Se llegó al acuerdo de crear un CD con un portafolio con algunas estrategias del modelo dimensiones del aprendizaje que les habían funcionado y otros elementos como los formatos de asistencia, tareas y hojas de planeación.

\section{Discusión y reflexiones finales}

Dado que la CPA trabaja sobre una base de aprendizaje organizacional, esto es, de aprendizaje colectivo que tenga impacto en la organización, al establecer la semejanza entre las dos escuelas estudiadas puede decirse que las profesoras en ambos casos cumplieron con las etapas del modelo propuesto de manera natural, al aprender, compartir, usar conocimiento, y difundirlo, tanto para el beneficio individual de su propia práctica como también de la organización escolar en términos académicos, a pesar de las condiciones de trabajo en que se desenvuelven.

El compartir la experiencia es un rasgo muy importante que caracterizó a las profesoras de ambas escuelas. Estas acciones de colaboración generan una sensación de comunidad, de comunicación y colaboración que rompen con el aislamiento del profesorado (Lortie, 1974), son muy afines a lo que aquí se entiende por liderazgo docente, que en ambas escuelas surge de manera espontánea, al dar voz a las profesoras con sus iniciativas, ideas, experiencias, creatividad y el reconocimiento al dominio personal. Adicionalmente se observa la forma en que las participantes se apropiaron del proceso y fueron modelando los productos esperados acorde a las necesidades que fueron identificadas como relevantes o prioritarias, en el entendido de que la agenda era dictada por ellas en la búsqueda de mejorar su desempeño docente. Desde esta perspectiva, se aprecia que, si bien en la bibliografia se señala como un factor de éxito el liderazgo escolar efectivo en la mejora escolar (Gurr y Day, 2014), en la documentación de estos casos se puede observar que, aunque idóneo, resulta no ser indispensable. En la medida en que los grupos docentes encuentren en el espacio de colaboración un sustento valioso para resolver problemas, la CPA tienen oportunidad de legitimarse como parte de las estructuras de las escuelas. 
http://doi.org/10.15359/ree.25-1.4

http://www.una.ac.cr/educare

educare@una.ac.cr

Las dos escuelas son diferentes en cuanto a su tamaño, estrato socioeconómico del alumnado, número de comunidades, profesoras involucradas en las CPA, los problemas y temas identificados, así como la disposición y liderazgos de las directoras. En la escuela Leona Vicario todas las maestras participaron en la CPA motivadas inicialmente por la directora, lo cual sugiere la ausencia de liderazgo distribuido (Harris y Jones, 2017), lo que conllevaría a una colegialidad artificial o balcanización (Hargreaves, 2005). Sin embargo, en la primera fase del aprendizaje organizacional, de forma voluntaria se crearon dos equipos de trabajo que desarrollaron el proceso de aprender y compartir en forma independiente y colectiva, mientras que en la escuela Vicente Guerrero no se contó con el apoyo de las directivas, y aún así hubo profesoras que participaron voluntariamente. Estas decidieron trabajar en la CPA espontáneamente y sin presiones de las directivas y de horario, lo que plantea la siguiente pregunta: ¿es posible que haya ocurrido un proceso de autoselección para integrar una comunidad en la escuela Vicente Guerrero? Otra de las diferencias fue que las profesoras de la escuela Leona Vicario, tanto las novatas como las expertas reconocieron haber aprendido mutuamente entre ellas, mientras que en la Escuela Vicente Guerrero las novatas reconocieron haber obtenido conocimiento de las expertas.

Creemos que el hecho de haber intentado en este estudio que las profesoras se pudieran organizar como CPA y participaran a través del ciclo de aprendizaje organizacional les brindó el espacio para ejercer su liderazgo; pero, sobre todo, las empoderó al reafirmar su capacidad de actuar como profesionales en el sentido de reflexionar sobre su práctica para generar y aplicar conocimiento a situaciones nuevas o problemáticas (Schön, 1983), de esta forma, la práctica profesional docente se recrea y mejora a partir de los nuevos aprendizajes logrados por medio de las CPA.

DuFour y Eaker (1998) han propuesto que las escuelas se conviertan en CPA que incluyan a los maestros y las maestras en todos los aspectos de la mejora de la escuela y enfatizan la importancia del liderazgo, argumentando que para lograr una verdadera CPA "los directores deben fomentar esta imagen del maestro como un líder y demostrar que consideran a los maestros como líderes compañeros en lugar de subordinados" (p. 198). En esta experiencia, en la escuela Vicente Guerrero fue notoria la indiferencia de la directora hacia las iniciativas de las profesoras, mientras que en la escuela Leona Vicario la directora animó a las maestras a participar en la CPA; sin embargo, las profesoras en las CPA trabajaron en aspectos de tipo académicos, enfocados a la enseñanza en el aula, situación que eventualmente podría conducir al aprendizaje organizacional en cuanto al proceso de su participación en las decisiones de la gestión escolar.

Cabe reiterar que las CPA también son espacios propicios para el desarrollo de aprendizajes para toda la comunidad educativa, en los cuales las directivas pudieran aprovechar las iniciativas, la capacidad de innovar y compartir conocimiento de las profesoras para reflexionar en conjunto acerca de situaciones y problemáticas de gestión que les atañen, así como en sus soluciones 
http://doi.org/10.15359/ree.25-1.4

y con ello generar conocimiento organizacional que permita el desarrollo sostenible de los centros escolares. Bailey Moreno et al. (2016) señalan que este proceso no es sencillo ni existen estrategias y procedimientos únicos, más bien plantean que"maestros y administradores en sus centros escolares establezcan sus propios proyectos y estrategias a partir de sus necesidades e intereses, y sean co-creadores de sus procesos de aprendizaje y cambio organizacional" (p.19).

Si consideramos al liderazgo distribuido como la capacidad de aprender y compartir conocimiento entre los profesorado y personal administrativo en un ambiente de colaboración, dada la dinámica y temáticas de trabajo de las CPA en estas dos escuelas, se puede concluir que la experiencia ayudó a las profesoras a crecer y a aprender, sin embargo, no se amplió el proceso de aprendizaje en interacción con los estratos directivos o administradores escolares. En México hay un camino importante que recorrer para que personal directiv consideren que la participación del profesorado puede enriquecer la toma de decisiones en la gestión escolar. Lattimer (2007) señala que los maestros y las maestras estarán a la altura de su potencial como líderes solo cuando el ambiente escolar apoye sus esfuerzos. En los casos aquí descritos fue notable observar la motivación de las profesoras para involucrarse en una CPA, de lo cual se puede inferir que es posible su creación en las escuelas, sin descartar las resistencias que ocurren cuando se implementan procesos de cambio, pero siempre con el apoyo directivo.

Estos procesos de colaboración en CPA y de liderazgo distribuido deberían constituirse como pilares de la escuela y ser incorporados en la cultura escolar, sin embargo, la estructura escolar mexicana en la que sigue prevaleciendo el aislamiento de los profesores y las profesoras, así como una gestión escolar unilateral, hace complicado que estos procesos ocurran. Aunado a las resistencias e incertidumbre para enfrentar y solucionar problemas desde nuevas visiones que brinda el liderazgo distribuido en contextos de colaboración que ofrece una CPA. Consideramos que los temas de liderazgo, colaboración y CPA deberán ser una parte esencial de la profesionalización docente desde etapas tempranas; para que esto suceda, es preciso que el conocimiento y práctica de estos temas se inicien curricularmente desde la educación formal del futuro personal docente en las escuelas formadoras y continúe durante el desarrollo de su práctica.

Aún haymuchoporhacereinvestigarenlalínea deliderazgodocenteyenlaimplementación de CPA en las escuelas primarias, particularmente en México, donde se han conducido escasos estudios. Existen dificultades por superar necesariamente, dado que la estructura organizacional imperante impide el establecimiento de espacios informales de profesores y profesoras que les permita compartir y usar el conocimiento de su práctica educativa, por lo que es importante estimularles compartir su experiencia con otros compañeros y compañeras. Cada organización debe buscar y encontrar, en sus miembros, a aquellas personas que tienen las soluciones o ideas para los problemas que necesitan ser resueltos. En México, el reto será crear nuevas estructuras y espacios como los descritos aquí. En las voluntades de los profesores, profesoras directivos e investigadores está la respuesta al cambio educativo en esta línea. 
http://doi.org/10.15359/ree.25-1.4

http://www.una.ac.cr/educare

educare@una.ac.cr

\section{Declaración de Material complementario}

Este artículo tiene disponible, como material complementario:

-La versión preprint del artículo en https://doi.org/10.5281/zenodo.3471967

\section{Referencias}

Argyris, C. (2001). Sobre el aprendizaje organizacional. Oxford: University Press.

Bailey Moreno, J., Flores Fahara, M. y González Rivera, P. E. (2016). Aprendizaje docente y reestructuración de sus creencias en los procesos de desarrollo profesional y gestión escolar. Revista Actualidades Investigativas en Educación, 16(3), 1-25. https://revistas.ucr. ac.cr/index.php/aie/article/view/25956

Boles, K.C. (1992). School restructuring by teachers: A study of the teaching project at the Edward Devotion School. Trabajo presentado en la Annual Meeting of the American Educational Research Association, San Francisco.

Carpenter, D. (2015). School culture and professional learning communities. International Journal of Educational Management, 29(5), 682-694. https://doi.org/10.1108/IJEM-04-2014-0046

Darling-Hammond, L. (1996). The quiet revolution: Rethinking teacher development. Educational Leadership, 53(6), 4-10. Recuperado de http://www.ascd.org/publications/educationalleadership/mar96/vol53/num06/toc.aspx

Gurr, D. y Day, C. (2014). Leading school successfully. En C. Day y D. Gurr (Eds.), Leading school successfully. Stories from the field (pp. 1-6). Routledge.

Day, C. y Harris, A. (2003). Teacher leadership, reflective practice and school improvement. En K. Leithwood y P. Hallinger (Eds.), Second international handbook of educational leadership and administration (pp. 957-977). Boston: Springer-Kluwer. https://doi.org/10.1007/97894-010-0375-9 32

DiBella, A. J. y Nevis, E. C. (1998). How organizations learn: An integrated strategy for building learning capability. Jossey-Bass.

DuFour, R.y Eaker, R. (1998). Professional learning communities at work: Best practices for enhancing student achievement. Association for Supervision and Curriculum Development.

Elizondo Huerta, A. (2010). La nueva escuela, I. Dirección, liderazgo y gestión escolar. Paidós.

Erlandson, D. A., Harris, E. L., Skipper, B. L. y Allen, S. D. (1993). Doing naturalistic inquiry: A guide to methods. Sage. 
http://doi.org/10.15359/ree.25-1.4 http://www.una.ac.cr/educare educare@una.ac.cr

Flores, E. y Flores, M. (2005). Public schools as learning communities: A multiple-case study of collaborative research in Northern Mexico. Trabajo presentado en la Annual Meeting of the American Educational Research Association. Montreal, Canadá.

Fullan, M. (2016). The new meaning of educational change (5. a ed.). Teachers College Press.

Gibbs, G. (2014). El análisis de datos cualitativos en investigación cualitativa. Morata.

Hargreaves, A. (2005). Profesorado, cultura y postmodernidad (Cambian los tiempos, cambia el profesorado). Morata.

Harris, A. (2003).Teacher leadership as distributed leadership: Heresy, fantasy or possibility? School Leadership \&Management, 23(3), 313-324. https://doi.org/10.1080/1363243032000112801

Harris, A. y Jones, M. S. (2017). Professional learning communities: A strategy for school and system improvement. Wales Journal of Education, 19(1), 16-38. https://doi.org/10.16922/ wje.19.1.2

Katzenmeyer, M. y Moller, G. (2009). Awakening the sleeping giant. Helping teachers develop as leaders. Corwin Press.

Lattimer, H. (2007). To help and not hinder. Educational Leadership, 65(1), 70-73. Recuperado de http://www.ascd.org/publications/educational-leadership/sept07/vol65/num01/ToHelp-and-Not-Hinder.aspx

Lincoln, Y. S y Guba, E. G. (1985). Naturalistic inquiry. Sage.

Lortie, D. C. (1974). Schoolteacher: A sociological study. University of Chicago Press.

Louis, K. S. y Kruse, S. D. (1995). Professionalism and community: Perspectives on reforming urban schools. Corwin Press.

Martínez-Cruz, E. y Moreno-Olivos, T. (2017). La colegialidad docente en la escuela primaria mexicana: Análisis de sus prácticas. Revista Electrónica Educare, 21(2), 1-21. https://doi. org/10.15359/ree.21-2.6

Marzano, R. J., Pickering, D. J., Arredondo, D. E., Blackburn, G. J., Brandt, R. S., Moffett, C. A., Paynter, D. E., Pollock, J. E. y Whisler, J. S. (2011). Dimensions of learning teacher's manual. ASCD.

Mason, J. (2018). Qualitative researching (3. ${ }^{\text {a }}$ ed.). Sage.

Merriam, S. B. (2015). Qualitative research. A guide to design and implementation. Jossey Bass.

Reeves, D. B. (2009). Leading change in your school: How to conquer myths, build commitment, and get results. ASCD. 
http://doi.org/10.15359/ree.25-1.4

http://www.una.ac.cr/educare

educare@una.ac.cr

Rosenholtz, S. (1989). Teacher s 'workplace: The social organization of schools. Longman.

Schön, D. A. (1983). The reflective practitioner: How professionals think in action. Basic Books.

Secretaría de Educación Pública. (7 de diciembre de 1982). Acuerdo 96. Que establece la organización y funcionamiento de las escuelas primarias. Diario Oficial de la Federación. http://dof.gob.mx/nota detalle.php?codigo=4784713\&fecha=07/12/1982

Senge, P. M. (2006). The fifth discipline: The art \& practice of the learning organization. Doubleday/ Currency.

Sepúlveda, F. y Aparicio, C. (2017). El desafío de los directores de escuelas chilenas: Liderando a partir de un enfoque instruccional hacia un enfoque distribuido. Revista Gestión de la Educación, 7(2), 1-19. Recuperado de https://revistas.ucr.ac.cr/index.php/gestedu/article/ view/30599

Spillane, J. P. (2006). Distributed leadership. Jossey-Bass.

Wasley, P. A. (1991). Teachers who lead: the rhetoric of reform and the realities of practice. Teachers College Press.

Wenger, E. (2001). Comunidades de práctica: Aprendizaje, significado e identidad. Paidós.

Yin, R. K. (2017). Case study research and applications: Design and methods. Sage. 